\title{
Association between Platelet-Specific Collagen Receptor Glycoprotein 6 Gene Variants, Selected Biomarkers, and Recurrent Pregnancy Loss in Korean Women
}

\author{
Hui Jeong An ${ }^{1,+}$, Eun Hee Ahn ${ }^{2,+}{ }^{,}$Jung Oh Kim ${ }^{1}$, Chang Soo Ryu ${ }^{1}$, Han Sung Park ${ }^{1}$, \\ Sung Hwan Cho ${ }^{1}$, Ji Hyang Kim ${ }^{2}$, Woo Sik Lee ${ }^{3}$, Jung Ryeol Lee ${ }^{4} \oplus$, Young Ran Kim ${ }^{2, *}$ \\ and Nam Keun Kim 1,*D \\ 1 Department of Biomedical Science, College of Life Science, CHA University, Seongnam 13488, Korea; \\ tody2209@naver.com (H.J.A.); jokim8505@gmail.com (J.O.K.); regis2040@nate.com (C.S.R.); \\ hahnsung@naver.com (H.S.P.); arana006@naver.com (S.H.C.) \\ 2 Department of Obstetrics and Gynecology, CHA Bundang Medical Center, School of Medicine, CHA \\ University, Seongnam 13488, Korea; bestob@chamc.co.kr (E.H.A.); bin0902@chamc.co.kr (J.H.K.) \\ 3 Fertility Center of CHA Gangnam Medical Center, CHA University, Seoul 061, Korea; wooslee@cha.ac.kr \\ 4 Department of Obstetrics and Gynecology, Seoul National University Bundang Hospital, \\ Seongnam 13620, Korea; leejrmd@snu.ac.kr \\ * $\quad$ Correspondence: happyiran@cha.ac.kr (Y.R.K.); nkkim@cha.ac.kr (N.K.K.); \\ Tel.: +82-31-780-5293 (Y.R.K.); +82-31-881-7137 (N.K.K.) \\ + These authors contributed equally to this work.
}

Received: 12 May 2020; Accepted: 25 July 2020; Published: 29 July 2020

\begin{abstract}
This paper investigates whether glycoprotein 6 (GP6) gene polymorphisms are a risk factor for recurrent pregnancy loss (RPL) in Korean women. Genotypes were determined by polymerase chain reaction-restriction fragment length polymorphism and real-time polymerase chain reaction amplification. We identified five polymorphisms in the GP6 gene: rs1654410 T>C, rs1671153 T>G, rs1654419 G>A, rs12610286 A>G, and rs1654431 G>A. GP6 rs1654410 CC was associated with decreased RPL risk (adjusted odds ratio $=0.292,95 \%$ confidence interval $=0.105-0.815, p=0.019$ ), and recessive genotypes were also significantly associated with decreased RPL risk (adjusted odds ratio $=0.348,95 \%$ confidence interval $=0.128-0.944, p=0.038$ ). GP6 rs1654419 GA was associated with decreased RPL risk (adjusted odds ratio $=0.607,95 \%$ confidence interval $=0.375-0.982, p=0.042$ ), and dominant genotypes were significantly associated with decreased RPL risk (adjusted odds ratio $=0.563,95 \%$ confidence interval $=0.358-0.885, p=0.013$ ). Altogether, the genotype frequencies of GP6 rs1654410 T>C and GP6 rs1654419 G>A were significantly different between RPL patients and control participants. Therefore, although GP6 polymorphisms may be useful as biomarkers of RPL, additional studies with heterogeneous cohorts are required to better understand the influence of GP6 and assess its performance as a biomarker.
\end{abstract}

Keywords: glycoprotein 6 (GP6); recurrent pregnancy loss; polymorphism; platelet; miRNA

\section{Introduction}

Recurrent pregnancy loss (RPL) is defined as two or more consecutive pregnancy losses [1]. Factors that contribute to the etiology of RPL include advanced maternal age, maternal anatomic anomalies, placental anomalies, chromosome abnormalities, endocrine dysfunction, antiphospholipid syndrome, hereditary thrombophilia, psychological trauma, and environmental factors, such as smoking, excessive alcohol consumption, and stress [2]. Moreover, the likelihood of pregnancy loss is $5 \%$ higher for women 
who suffer a miscarriage during their first pregnancy than for healthy subjects [3]. Although many factors that contribute to the etiology of RPL have been identified, the underlying cause remains unknown in the majority of cases. RPL is associated with immune disorders, blood coagulation, and angiogenesis. These factors are related to hemostasis, which in turn contributes to platelet activation. Collagen is a platelet activator that plays an important role in the vascular endothelium and vascular wall. Platelets have two major receptors for collagen: integrins, which play a role in platelet aggregation, and glycoprotein 6 (GP6), which is primarily involved in signal transduction and platelet activation [4].

GP6 is an immunoglobulin-like collagen receptor that is exclusively expressed in platelets and megakaryocytes and is essential for cell activation in matrix proteins. GP6 is the product of the GP6 gene, which is located on chromosome 19 (19q13.4) [5]. Since the identification and analysis of the GP6 gene in the 1990s, a number of single nucleotide polymorphisms (SNPs) have been identified [6].

GP6 is essential for integrin activation, the formation of stable attachments, and subsequent signaling and platelet aggregation in both in vitro and in vivo studies [4]. The GP6 is a receptor for collagen and plays a critical role in collagen-induced platelet aggregation and thrombus formation [7]. In fact, several GP6 polymorphisms (rs1671153, rs1654419, and rs1613662) are associated with an increased risk of platelet aggregation $[8,9]$. The precise mechanisms regulating GP6 gene molecular variations and aggregation function are largely unknown; however, some studies have suggested that the genetic polymorphisms in GP6 may influence receptor density and platelet function [10,11]. The aim of the present study was twofold: to evaluate the genetic variability of the GP6 gene in control and patients with RPL, and to carry out association analyses of GP6 with two, three, or four pregnancy losses. Specifically, we investigated the association between RPL and five GP6 polymorphisms (rs1654410 T>C, rs1671153 T>G, rs1654419 $\mathrm{G}>\mathrm{A}, \mathrm{rs} 12610286 \mathrm{~A}>\mathrm{G}$, and rs1654431 G>A) in a population of Korean women. Sticky platelet syndrome (SPS) is the second most common thrombophilia that causes recurrent spontaneous abortions or fetal loss syndrome [12,13]. It was found that there was a significant association between GP6 gene polymorphism and thromboembolic events in SPS patients [14]. Sokol et al. [9] has found that some polymorphisms of GP6 (including rs1654410, rs1671153, rs1654419, rs12610286, and rs1654431) represent a risk factor in patients with SPS and fetal loss. Considering the critical role of GP6 in platelet coagulant activity, the polymorphisms in GP6 may influence pregnancy loss. We demonstrated that GP6 rs1654410C > T, GP6 rs1654419 G>A, and GP6 rs1654431 G>A SNPs were associated with RPL in this patient cohort.

\section{Materials and Methods}

\subsection{Participants}

Blood samples were collected from 388 patients with RPL (mean age \pm SD, $33.20 \pm 4.54$ years) and 219 control participants (mean age \pm SD, $32.75 \pm 3.84$ years). The RPL patients were recruited from the Department of Obstetrics and Gynecology or Fertility Center of the CHA Bundang Medical Center in Seongnam between March 1999 and February 2010. The women in the control group were also recruited from the CHA Bundang Medical Center and met the following criteria: pregnant, regular menstrual cycles, a history of at least one naturally conceived pregnancy, no history of pregnancy loss, and karyotype 46, XX. The Institutional Review Board of the CHA Bundang Medical Center approved the study, and all patients gave written informed consent. The IRB number for this study was BD2010-123D. All patients had suffered a minimum of two consecutive spontaneous miscarriages, and blood samples were taken based on human chorionic gonadotropin (hCG) levels prior to 20 weeks gestation. The average gestational age was $7.32 \pm 2.05$ weeks. Pregnancy loss was diagnosed with human chorionic gonadotropin (hCG) testing, ultrasound, and/or physical examination before 20 weeks of gestational age. None of the participants had a history of smoking or alcohol use. Patients with recurrent pregnancy loss due to anatomic, hormonal, chromosomal, infectious, autoimmune, or thrombotic causes were excluded from the study. All study protocols were abided by the recommendations of the Declaration of Helsinki, and written informed consent was obtained from all study participants. 


\subsection{Genotyping}

Genomic DNA was extracted from anticoagulated peripheral blood samples using a G-DEX ${ }^{\mathrm{TM}}$ Genomic DNA Extraction Kit for blood (iNtRON Biotechnology, Seongnam, Korea) [15]. Five GP6 (SNPs) were selected using the human genome SNP database (dbSNP, http://www.ncbi.nlm.nih.gov/snp).

For RFLP analysis of the SNPs, the PCR products for GP6T >C rs1654410, GP6T >G, rs1671153, GP6G>Ars1654419 were digested with the restriction enzymes Mbo II, Hph I, and Cse I, respectively. To confirm the three SNPs and validate the RFLP results, $10-20 \%$ of the samples were randomly selected, used for a second round of PCR, and analyzed by DNA sequencing using an automatic ABI3730xL DNA analyzer (Applied Biosystems, Forster City, CA, USA). Samples were genotyped by real-time polymerase chain reaction (PCR) using a qPCR kit with the primers listed in Supplementary Table S1. The conditions for the five polymorphisms (rs1654410 T>C, rs1671153 T>G, rs1654419 G>A, rs12610286 A>G, and rs1654431 G>A) are also listed in Supplementary Table S1.

\subsection{Assessment of Plasma Plasminogen Activator Inhibitor-1 (PAI-1), Homocysteine, Total Cholesterol, Uric Acid, and Blood Coagulation Status}

Plasma PAI-1, homocysteine, total cholesterol, and uric acid were measured in blood collected from RPL patients. Plasma was separated by centrifuging whole blood samples at $1000 \times g$ for $15 \mathrm{~min}$. PAI- 1 levels were determined using a human serpin E1/PAI-1 immunoassay (R\&D Systems, Minneapolis, MN, USA). Uric acid and total cholesterol were measured using commercially available enzymatic colorimetric tests (Roche Diagnostics, GmbH, Mannheim, Germany). Homocysteine was measured using a fluorescence polarization immunoassay using the Abbott IMx analyzer (Abbott Laboratories, Abbott Park, IL, USA).

\subsection{Statistical Analysis}

Differences in the frequency of GP6 polymorphisms between the control and patient groups were assessed using Fisher's exact test and a logistic regression model. Odds ratios (ORs), adjusted odds ratios (AORs), and 95\% confidence intervals (CIs) were used to examine the association between GP6 polymorphisms and RPL risk. The data are presented as mean \pm standard deviation (SD) for continuous variables or as percentages for categorical variables. Statistical analyses were carried out using MedCalc version 12.1.4 (MedCalc Software bvba, Mariakerke, Belgium) or GraphPad Prism 4.0 (GraphPad Software, Inc., San Diego, CA, USA). The HAPSTAT program (v.3.0, www.bios.unc.edu/ \{\}lin/hapstat/) was used with a strong synergistic effect to estimate the frequency of polymorphic haplotypes, $p$-values of $<0.05$ were considered statistically significant. The false discovery rate (FDR) was also used to adjust for multiple comparisons; associations with an FDR-corrected $p$-value of $<0.05$ were considered statistically significant [16]. Genetic interaction analysis was performed with the open-source multifactor dimensionality reduction (MDR) software package (v.2.0) available from www.epistasis.org. The MDR method consists of two main steps [17-19]. During ANOVA analysis, the Kruskal-Wallis test was used for small sample sizes and/or when the P-value of Levene's test was less than 0.05 (Figure 1).

\section{Results}

The clinical profiles and demographic characteristics of RPL patients and control participants are presented in Table 1. The mean ages of RPL and control patients were $32.75 \pm 3.84$ and $33.20 \pm 4.54$ years, respectively. When comparing the RPL patients with the control participants, the RPL patients had significantly higher platelet counts (PLT), prothrombin times (PT), activated partial thromboplastin times (aPTT), luteinizing hormone $(\mathrm{LH})$ levels $(\mathrm{mIU} / \mathrm{mL}$, mean $\pm \mathrm{SD})$, and estradiol (E2) levels $(\mathrm{pg} / \mathrm{mL}$, mean $\pm \mathrm{SD}$ ) (all $p<0.05$; Table 1). However, many of the factors measured here undergo changes during the course of pregnancy.

The genotype frequencies and ORs of the GP6 polymorphisms rs1654410 T>C, rs1671153 T>G, rs1654419 G > A, rs12610286 A > G, and rs1654431 G > A in control and RPL patients are listed in Table 2. All of the genotype frequencies that were analyzed showed polymorphisms and occurred in Hardy-Weinberg 
equilibrium (HWE) in both groups. The map of the GP6 gene is shown in Figure S1. We found a significant association between GP6 polymorphisms and three or more RPLs: GP6 rs1654410 T>C (TT versus CC: $\mathrm{AOR}=0.292 ; 95 \% \mathrm{CI}=0.105-0.815 ; p=0.019),(\mathrm{TT}+\mathrm{TC}$ versus $\mathrm{CC}: \mathrm{AOR}=0.348 ; 95 \%$ $\mathrm{CI}=0.128-0.944 ; p=0.038) . \mathrm{GP6}$ rs1654419 G>A (GG versus GA: AOR =0.607; 95\% CI = 0.375-0.982; $p=0.042)$, (GG versus GA + AA: AOR $=0.563 ; 95 \% \mathrm{CI}=0.358-0.885 ; p=0.013)$, and $\mathrm{GP} 6 \mathrm{rs} 1654431 \mathrm{G}>\mathrm{A}$ (GG versus GA: $\mathrm{AOR}=1.663 ; 95 \% \mathrm{CI}=1.007-2.746 ; p=0.047),(\mathrm{GG}$ versus $\mathrm{GA}+\mathrm{AA}: \mathrm{AOR}=1.746 ; 95 \%$ $\mathrm{CI}=1.082-2.818 ; p=0.022)$ compared with the control group.

Table 1. Clinical variables in control participants and RPL patients.

\begin{tabular}{|c|c|c|c|}
\hline Characteristics & Controls $(n=219)$ & RPL $(n=388)$ & $p$ \\
\hline Age (years) & $32.75 \pm 3.84$ & $33.20 \pm 4.54$ & 0.211 \\
\hline BMI $\left(\mathrm{kg} / \mathrm{m}^{2}\right)$ & $21.63 \pm 3.44$ & $19.77 \pm 6.83$ & 0.291 \\
\hline Previous pregnancy losses $(n)$ & N/A & $3.28 \pm 1.83$ & \\
\hline Live births $(n)$ & $1.71 \pm 0.58$ & N/A & \\
\hline Mean gestational age (weeks) & $39.14 \pm 1.56$ & $7.32 \pm 2.05$ & $<0.0001$ \\
\hline Homocysteine $(\mu \mathrm{mol} / \mathrm{L})$ & $35.98 \pm 4.13$ & $37.30 \pm 3.36$ & \\
\hline Total cholesterol (mg/dL) & $\mathrm{N} / \mathrm{A}$ & $187.73 \pm 49.41$ & \\
\hline Uric acid (mg/dL) & N/A & $3.80 \pm 0.83$ & 0.357 \\
\hline PLT $\left(10^{3} / \mu \mathrm{L}\right)$ & $242.11 \pm 61.86$ & $255.43 \pm 59.22$ & 0.034 \\
\hline PT (sec) & $0.84 \pm 0.09$ & $0.98 \pm 0.09$ & $<0.0001$ \\
\hline PAI-1 (ng/mL) & NA & $10.37 \pm 5.70$ & \\
\hline $\mathrm{aPTT}(\mathrm{sec})$ & $33.05 \pm 3.08$ & $32.23 \pm 4.32$ & 0.203 \\
\hline BUN (mg/dL, mean \pm SD) & N/A & $9.98 \pm 2.76$ & \\
\hline Creatinine $(\mathrm{mg} / \mathrm{dL}$, mean $\pm \mathrm{SD})$ & N/A & $0.72 \pm 0.12$ & \\
\hline FSH $(\mathrm{mIU} / \mathrm{mL}$, mean $\pm \mathrm{SD})$ & $8.13 \pm 2.80$ & $7.51 \pm 10.52$ & 0.546 \\
\hline $\mathrm{LH}(\mathrm{mIU} / \mathrm{mL}$, mean $\pm \mathrm{SD})$ & $3.38 \pm 1.87$ & $6.29 \pm 12.08$ & 0.012 \\
\hline $\mathrm{E} 2(\mathrm{pg} / \mathrm{mL}$, mean $\pm \mathrm{SD})$ & $26.00 \pm 14.94$ & $35.70 \pm 29.45$ & 0.001 \\
\hline
\end{tabular}

Note: RPL, recurrent pregnancy loss; BMI, body mass index; PLT, platelet count; PT, prothrombin time; PAI-1, plasminogen activator inhibitor-1; aPTT, activated par tial thromboplastin time; BUN, blood urea nitrogen; FSH, follicle-stimulating hormone; LH, luteinizing hormone; E2, estradiol.

When the number of RPLs was increased to four or more, only two GP6 variants remained associated with RPL risk: GP6 rs1654419 G>A (GG versus GA: AOR = 0.506; 95\% CI = 0.273-0.940; $p=0.031$ ), (GG versus $\mathrm{GA}+\mathrm{AA}: \mathrm{AOR}=0.473 ; 95 \% \mathrm{CI}=0.265-0.846 ; p=0.012)$ and $\mathrm{GP} 6$ rs1654431 G>A (GG versus GA: $\mathrm{AOR}=1.967 ; 95 \% \mathrm{CI}=1.052-3.677 ; p=0.034),(\mathrm{GG}$ versus $\mathrm{GA}+\mathrm{AA}: \mathrm{AOR}=1.856 ; 95 \% \mathrm{CI}=1.013-3.400$; $p=0.045)$. However, after FDR-p correction, the association was not statistically significant.

To further analyze the association of the five GP6 polymorphisms (rs1654410 T>C, rs1671153 T>G, rs1654419 G>A, rs12610286 A > G, and rs1654431 G>A) with RPL, we performed a combinatorial analysis (Table 3). Analysis of the five polymorphisms demonstrated that the frequency of GP6 polymorphic genotype combinations was significantly higher in RPL patients compared to control participants. The genotype combination analysis of four GP6 polymorphisms is also presented in Table 3 as GP6 rs1654410/GP6 rs1671153 TT/TT (AOR =0.333 95\% CI =0.140-0.792; $p=0.013)$. Likewise, the two site combination genotypes GP6 rs1654410/GP6 rs1654419 TT/AA (AOR =0.276 95\% CI =0.113-0.674; $p=0.005)$ and TT/GA (AOR $=0.20295 \% \mathrm{CI}=0.050-0.813 ; p=0.024)$ also demonstrated significant associations with RPL.

Since environmental factors are known to contribute to RPL, we also investigated the interaction of clinical characteristics and GP6 genotypes. Logistic regression analysis was used for the association of each SNP with RPL risk adjusted by environmental factors. Table 4 shows that the GP6 rs1654419 GG + GA genotype increased RPL risk when the following characteristics were present: platelet count of $\geq 242.11 \times 10^{3}(\mathrm{AOR}=4.461 ; 95 \% \mathrm{CI}=2.234-8.905)$, follicle-stimulating hormone $(\mathrm{FSH})$ level $<8.13 \mathrm{mIU} / \mathrm{mL}(\mathrm{AOR}=2.716 ; 95 \% \mathrm{CI}=1.458-5.057)$, and $\mathrm{E} 2$ level $<26.00 \mathrm{pg} / \mathrm{mL}(\mathrm{AOR}=2.322 ; 95 \% \mathrm{CI}$ $=1.134-4.755)$. Thus, the GP6 rs1654419 genotype may present an increased risk of RPL. However, these factors measured here undergo changes in the course of pregnancy. 
Table 2. Genotype frequencies of glycoprotein 6 (GP6) polymorphisms in the control and RPL patients.

\begin{tabular}{|c|c|c|c|c|c|c|c|c|c|c|c|c|c|}
\hline Genotypes & $\begin{array}{l}\text { Controls } \\
(n=219)\end{array}$ & $\begin{array}{c}\text { PL } \geq 2 \\
(n=388)\end{array}$ & AOR $(95 \% \mathrm{CI})$ & $p$ & FDR-P & $\begin{array}{l}\text { Controls } \\
(n=219)\end{array}$ & $\begin{array}{c}\text { PL } \geq 3 \\
(n=138)\end{array}$ & AOR $(95 \% \mathrm{CI})$ & $p$ & FDR-P & $\begin{array}{c}\text { PL } \geq 4 \\
(n=74)\end{array}$ & AOR $(95 \% \mathrm{CI})$ & $p$ \\
\hline \multicolumn{14}{|l|}{ GP6 rs1654410T $>C$} \\
\hline TT & $88(40.2)$ & $189(48.7)$ & 1.000 (reference) & & & $88(40.2)$ & $69(50.0)$ & 1.000 (reference) & & & $37(50.0)$ & 1.000 (reference) & \\
\hline TC & $108(49.3)$ & $176(45.4)$ & $1.218(0.737-2.012)$ & 0.432 & 0.781 & $108(49.3)$ & $64(46.4)$ & $0.760(0.488-1.183)$ & 0.223 & 0.309 & $34(45.9)$ & $0.760(0.440-1.312)$ & 0.325 \\
\hline $\mathrm{CC}$ & $23(10.5)$ & $23(5.9)$ & $1.285(0.693-2.385)$ & 0.446 & 0.511 & $23(10.5)$ & $5(3.6)$ & $0.292(0.105-0.815)$ & 0.019 & 0.095 & $3(4.1)$ & $0.338(0.094-1.209)$ & 0.095 \\
\hline Dominant (TT vs. TC $+\mathrm{CC}$ ) & & & $1.236(0.766-1.995)$ & 0.355 & 0.627 & & & $0.684(0.444-1.054)$ & 0.085 & 0.142 & & $0.695(0.408-1.185)$ & 0.182 \\
\hline Recessive (TT + TC vs. CC) & & & $1.126(0.672-1.888)$ & 0.641 & 0.641 & & & $0.348(0.128-0.944)$ & 0.038 & 0.190 & & $0.395(0.114-1.368)$ & 0.143 \\
\hline HWE $P$ & 0.423 & 0.302 & & & & & & & & & & & \\
\hline \multicolumn{14}{|l|}{ GP6 rs1671153 T>G } \\
\hline TT & $107(48.9)$ & $216(55.7)$ & 1.000 (reference) & & & $107(48.9)$ & $74(53.6)$ & 1.000 (reference) & & & $37(50.0)$ & 1.000 (reference) & \\
\hline TG & 98 (44.7) & 155 (39.9) & $0.788(0.558-1.113)$ & 0.176 & 0.781 & $98(44.7)$ & $55(39.9)$ & $0.826(0.528-1.291)$ & 0.401 & 0.401 & $29(39.2)$ & $0.879(0.501-1.543)$ & 0.653 \\
\hline GG & $14(6.4)$ & $17(4.4)$ & $0.625(0.295-1.324)$ & 0.220 & 0.511 & $14(6.4)$ & $9(6.5)$ & $1.039(0.421-2.567)$ & 0.934 & 0.934 & $8(10.8)$ & $1.905(0.720-5.042)$ & 0.194 \\
\hline Dominant (TT vs. TG + GG) & & & $0.770(0.551-1.076)$ & 0.125 & 0.625 & & & $0.852(0.554-1.310)$ & 0.465 & 0.465 & & $0.994(0.584-1.693)$ & 0.983 \\
\hline Recessive (TT + TG vs. GG) & & & $0.693(0.334-1.439)$ & 0.325 & 0.542 & & & $1.153(0.480-2.768)$ & 0.751 & 0.751 & & $2.051(0.810-5.195)$ & 0.130 \\
\hline HWE $P$ & 0.173 & 0.097 & & & & & & & & & & & \\
\hline \multicolumn{14}{|l|}{ GP6 rs1654419 G>A } \\
\hline GG & $122(55.7)$ & $262(67.5)$ & 1.000 (reference) & & & $122(55.7)$ & $96(69.6)$ & 1.000 (reference) & & & $54(73.0)$ & 1.000 (reference) & \\
\hline GA & 77 (35.2) & $106(27.3)$ & $0.920(0.580-1.461)$ & 0.625 & 0.781 & $77(35.2)$ & $36(26.1)$ & $0.607(0.375-0.982)$ & 0.042 & 0.118 & $17(23.0)$ & $0.506(0.273-0.940)$ & 0.031 \\
\hline AA & $20(9.1)$ & $20(5.2)$ & $1.294(0.700-2.394)$ & 0.511 & 0.511 & $20(9.1)$ & $6(4.3)$ & $0.392(0.151-1.018)$ & 0.055 & 0.114 & $3(4.1)$ & $0.345(0.098-1.213)$ & 0.097 \\
\hline Dominant (GG vs. GA + AA) & & & $1.006(0.653-1.551)$ & 0.878 & 0.878 & & & $0.563(0.358-0.885)$ & 0.013 & 0.055 & & $0.473(0.265-0.846)$ & 0.012 \\
\hline Recessive (GG + GA vs. AA) & & & $1.355(0.774-2.372)$ & 0.288 & 0.542 & & & $0.464(0.181-1.189)$ & 0.110 & 0.270 & & $0.427(0.123-1.486)$ & 0.181 \\
\hline HWE $P$ & 0.085 & 0.642 & & & & & & & & & & & \\
\hline \multicolumn{14}{|l|}{$G P 6$ rs12610286 A>G } \\
\hline AA & $118(53.9)$ & $202(52.1)$ & 1.000 (reference) & & & $118(53.9)$ & $64(46.4)$ & 1.000 (reference) & & & $31(41.9)$ & 1.000 (reference) & \\
\hline AG & $90(41.1)$ & $148(38.1)$ & $0.979(0.691-1.387)$ & 0.905 & 0.905 & $90(41.1)$ & $61(44.2)$ & $1.305(0.832-2.049)$ & 0.247 & 0.309 & $38(51.4)$ & $1.725(0.987-3.013)$ & 0.056 \\
\hline GG & $11(5.0)$ & $38(9.8)$ & $2.025(0.995-4.120)$ & 0.052 & 0.260 & $11(5.0)$ & $13(9.4)$ & $2.106(0.888-4.994)$ & 0.091 & 0.114 & $5(6.8)$ & $1.670(0.538-5.187)$ & 0.375 \\
\hline Dominant (AA vs. AG + GG) & & & $1.088(0.780-1.517)$ & 0.621 & 0.776 & & & $1.384(0.899-2.129)$ & 0.140 & 0.175 & & $1.689(0.986-2.895)$ & 0.056 \\
\hline Recessive (AA + AG vs. GG) & & & $2.006(1.001-4.018)$ & 0.050 & 0.250 & & & $1.822(0.787-4.219)$ & 0.162 & 0.270 & & $1.267(0.423-3.803)$ & 0.673 \\
\hline HWE $P$ & 0.238 & 0.160 & & & & & & & & & & & \\
\hline \multicolumn{14}{|l|}{ GP6 rs1654431 G>A } \\
\hline GG & $79(36.1)$ & $154(39.7)$ & 1.000 (reference) & & & $79(36.1)$ & $34(24.6)$ & 1.000 (reference) & & & $18(24.3)$ & 1.000 (reference) & \\
\hline GA & $104(47.5)$ & $178(45.9)$ & $0.875(0.608-1.259)$ & 0.472 & 0.781 & $104(47.5)$ & $74(53.6)$ & $1.663(1.007-2.746)$ & 0.047 & 0.118 & $45(60.8)$ & $1.967(1.052-3.677)$ & 0.034 \\
\hline AA & $36(16.4)$ & $56(14.4)$ & $0.774(0.468-1.279)$ & 0.318 & 0.511 & $36(16.4)$ & $30(21.7)$ & $1.772(0.932-3.369)$ & 0.081 & 0.114 & $11(14.9)$ & $1.391(0.582-3.325)$ & 0.458 \\
\hline Dominant (GG vs. GA + AA) & & & $0.856(0.608-1.207)$ & 0.376 & 0.627 & & & $1.746(1.082-2.818)$ & 0.022 & 0.055 & & $1.856(1.013-3.400)$ & 0.045 \\
\hline Recessive (GG + GA vs. AA) & & & $0.833(0.526-1.319)$ & 0.436 & 0.545 & & & $1.309(0.757-2.263)$ & 0.336 & 0.420 & & $0.860(0.412-1.799)$ & 0.689 \\
\hline HWE $P$ & 0.857 & 0.694 & & & & & & & & & & & \\
\hline
\end{tabular}

Note: AORs were adjusted for age of participants. RPL, recurrent pregnancy loss; AOR, adjusted odds ratio; CI, confidence interval; FDR-P, false discovery rate-adjusted $p$-value. 
Table 3. Genotype combinations of GP6 polymorphisms in control participants and (RPL) patients.

\begin{tabular}{|c|c|c|c|c|c|}
\hline Genotypes & Controls $(n=219)$ & RPL Patients $(n=388)$ & AOR (95\% CI) & $p^{a}$ & $F D R-P^{b}$ \\
\hline $\mathrm{TT} / \mathrm{TT}$ & $53(24.2)$ & $107(27.6)$ & 1.000 (reference) & & \\
\hline $\mathrm{CC} / \mathrm{TT}$ & $15(6.8)$ & $10(2.6)$ & $0.333(0.140-0.792)$ & 0.013 & 0.039 \\
\hline $\mathrm{CC} / \mathrm{GG}$ & $2(0.9)$ & $1(0.3)$ & $0.254(0.022-2.873)$ & 0.268 & 0.402 \\
\hline \multicolumn{6}{|c|}{ GP6 rs1654410/GP6 rs1654419 } \\
\hline $\mathrm{CC} / \mathrm{GG}$ & $15(6.8)$ & $19(4.9)$ & $0.662(0.307-1.430)$ & 0.294 & 0.725 \\
\hline CC/GA & $7(3.2)$ & $3(0.8)$ & $0.202(0.050-0.813)$ & 0.024 & 0.048 \\
\hline CC/AA & $1(0.5)$ & $1(0.3)$ & $0.443(0.027-7.270)$ & 0.569 & 0.569 \\
\hline \multicolumn{6}{|c|}{ GP6 rs1654410/GP6 rs12610286 } \\
\hline TT/AA & $48(21.9)$ & $100(25.8)$ & 1.000 (reference) & & \\
\hline TT/GG & $35(16.0)$ & $74(19.1)$ & 1.000 (reference) & & \\
\hline TT/GA & $57(26.0)$ & $78(20.1)$ & $0.640(0.377-1.087)$ & 0.099 & 0.396 \\
\hline $\mathrm{CC} / \mathrm{GG}$ & $10(4.6)$ & $6(1.5)$ & $0.282(0.094-0.852)$ & 0.025 & 0.100 \\
\hline \multicolumn{6}{|c|}{ GP6 rs1671153/GP6 rs1654419 } \\
\hline $\mathrm{TT} / \mathrm{GG}$ & $73(33.3)$ & $142(36.6)$ & 1.000 (reference) & & \\
\hline TG/AA & $12(5.5)$ & $10(2.6)$ & $0.391(0.157-0.974)$ & 0.044 & 0.132 \\
\hline GG/GA & $6(2.7)$ & $5(1.3)$ & $0.444(0.131-1.511)$ & 0.194 & 0.291 \\
\hline \multicolumn{6}{|c|}{ GP6 rs1671153/GP6 rs12610286 } \\
\hline TT/AA & $53(24.2)$ & $122(31.4)$ & 1.000 (reference) & & \\
\hline TT/AG & $52(23.7)$ & $66(17.0)$ & $0.556(0.342-0.905)$ & 0.018 & 0.072 \\
\hline TG/AG & $44(20.1)$ & $59(15.2)$ & $0.604(0.362-1.007)$ & 0.053 & 0.106 \\
\hline $\mathrm{GA} / \mathrm{AG}$ & $11(5.0)$ & $4(1.0)$ & $0.181(0.055-0.595)$ & 0.005 & 0.015 \\
\hline \multicolumn{6}{|c|}{ GP6 rs1654419/GP6 rs1654431 } \\
\hline GG/GG & $58(26.5)$ & $92(23.7)$ & 1.000 (reference) & & \\
\hline $\mathrm{AA} / \mathrm{AA}$ & $13(5.9)$ & 49 (12.6) & $2.203(1.091-4.447)$ & 0.028 & 0.112 \\
\hline AA/AA & $7(3.2)$ & $17(4.4)$ & $1.535(0.600-3.931)$ & 0.371 & 0.543 \\
\hline \multicolumn{6}{|c|}{ GP6 rs12610286/GP6 rs1654431 } \\
\hline AA/GG & $44(20.1)$ & $78(20.2)$ & 1.000 (reference) & & \\
\hline GG/AA & $8(3.7)$ & $34(8.8)$ & $2.373(1.004-5.608)$ & 0.049 & 0.196 \\
\hline AG/GA & $51(23.3)$ & $62(16.0)$ & $0.685(0.406-1.156)$ & 0.157 & 0.314 \\
\hline
\end{tabular}

Note: RPL, recurrent pregnancy loss; AOR, adjusted odds ratio; CI, confidence interval; a, Fisher's exact test; b, FDR-adjusted $p$ value.

Haplotype analyses were conducted to further assess the association of RPL occurrence with the five polymorphisms (Supplementary Table S2). Several haplotypes were found to be significantly associated with the incidence of RPL after FDR correction. Furthermore, the results from genotype combination analyses were significant for each allele, and these results were related to RPL incidence (Supplementary Table S3).

When the haplotype analysis was limited to four polymorphisms, we found that RPL was significantly associated with each allele (Supplementary Table S4). Limiting the haplotype analysis to three polymorphisms revealed that three variants (GP6 rs1654410, GP6 rs1671153, GP6 rs1654419) were the leading genetic risk factors related to RPL (Supplementary Table S5). 
Table 4. Interaction of RPL incidence and environmental factors.

\begin{tabular}{|c|c|c|c|c|c|c|c|c|c|c|}
\hline \multirow[t]{2}{*}{ Characteristics } & \multicolumn{2}{|c|}{ GP6 rs1654410 (TT vs. TC + CC) } & \multicolumn{2}{|c|}{ GP6 rs1671153 (TT vs. TG + GG) } & \multicolumn{2}{|c|}{ GP6 1654419 (GG + GA vs. AA) } & \multicolumn{2}{|c|}{ GP6 rs12610286 (AA + AG vs. GG) } & \multicolumn{2}{|c|}{ GP6 rs1654431 (GG + GA vs. AA) } \\
\hline & AOR $(95 \% \mathrm{CI}) *$ & $p$ & AOR $(95 \% \mathrm{CI}) *$ & $p$ & AOR $(95 \% \mathrm{CI}) *$ & $p$ & AOR $(95 \% \mathrm{CI}) *$ & $p$ & AOR $(95 \% \mathrm{CI}) *$ & $p$ \\
\hline Age & & & & & & & & & & \\
\hline$<32$ & $1.389(0.810-2.383)$ & 0.233 & $0.944(0.551-1.617)$ & 0.833 & $1.672(0.950-2.944)$ & 0.075 & $0.888(0.518-1.520)$ & 0.664 & $1.291(0.747-2.229)$ & 0.360 \\
\hline$\geq 32$ & $0.789(0.516-1.207)$ & 0.274 & $1.280(0.835-1.961)$ & 0.258 & $1.151(0.738-1.795)$ & 0.536 & $0.908(0.595-1.384)$ & 0.653 & $1.156(0.749-1.783)$ & 0.513 \\
\hline BMI & & & & & & & & & & \\
\hline$<25 \mathrm{~kg} / \mathrm{m}^{2}$ & $1.325(0.798-2.200)$ & 0.277 & $0.821(0.495-1.362)$ & 0.445 & $0.860(0.520-1.423)$ & 0.558 & $1.320(0.795-2.193)$ & 0.284 & $0.784(0.463-1.328)$ & 0.365 \\
\hline $\begin{array}{c}\geq 25 \mathrm{~kg} / \mathrm{m}^{2} \\
\text { Platelet }\end{array}$ & $0.434(0.099-1.893)$ & 0.267 & $1.433(0.365-5.623)$ & 0.606 & $2.398(0.544-10.571)$ & 0.248 & $0.487(0.118-2.006)$ & 0.319 & $1.245(0.307-5.041)$ & 0.759 \\
\hline$<242.11 \times 10^{3}$ cell & $0.908(0.498-1.655)$ & 0.752 & $1.078(0.586-1.981)$ & 0.810 & $1.056(0.578-1.931)$ & 0.859 & $0.841(0.462-1.532)$ & 0.572 & $1.294(0.710-2.356)$ & 0.400 \\
\hline $\begin{array}{c}\geq 242.11 \times 10^{3} \text { cell } \\
\text { PT }\end{array}$ & $1.108(0.622-1.973)$ & 0.728 & $1.267(0.711-2.258)$ & 0.422 & $4.461(2.234-8.905)$ & 0.0001 & $0.691(0.389-1.225)$ & 0.205 & $1.184(0.657-2.133)$ & 0.575 \\
\hline$\geq 0.84 \mathrm{~s}$ & $1.447(0.314-6.666)$ & 0.635 & $0.596(0.114-3.127)$ & 0.541 & $1.897(0.389-9.251)$ & 0.429 & $0.335(0.056-2.006)$ & 0.231 & $0.803(0.170-3.782)$ & 0.781 \\
\hline $\begin{array}{l}<0.84 \mathrm{~s} \\
\mathrm{aPTT}\end{array}$ & $1.208(0.533-2.740)$ & 0.651 & $0.630(0.274-1.448)$ & 0.277 & $0.696(0.306-1.582)$ & 0.387 & $0.844(0.372-1.913)$ & 0.684 & $0.761(0.314-1.843)$ & 0.545 \\
\hline$<33.05 \mathrm{~s}$ & $1.074(0.486-2.370)$ & 0.861 & $0.598(0.265-1.350)$ & 0.216 & $1.272(0.567-2.852)$ & 0.559 & $0.963(0.435-2.132)$ & 0.926 & $1.061(0.463-2.432)$ & 0.890 \\
\hline $\begin{array}{c}\geq 33.05 \mathrm{~s} \\
\text { FSH }\end{array}$ & $1.754(0.664-4.630)$ & 0.257 & $0.723(0.272-1.924)$ & 0.516 & $0.915(0.349-2.396)$ & 0.856 & $0.781(0.299-2.043)$ & 0.615 & $0.711(0.259-1.950)$ & 0.507 \\
\hline$<8.13 \mathrm{mIU} / \mathrm{mL}$ & $1.013(0.579-1.772)$ & 0.965 & $0.833(0.479-1.447)$ & 0.516 & $2.716(1.458-5.057)$ & 0.002 & $0.685(0.393-1.192)$ & 0.181 & $0.950(0.531-1.700)$ & 0.862 \\
\hline $\begin{array}{c}\geq 8.13 \mathrm{mIU} / \mathrm{mL} \\
\mathrm{LH}\end{array}$ & $0.906(0.382-2.150)$ & 0.822 & $0.672(0.283-1.595)$ & 0.367 & $0.921(0.381-2.226)$ & 0.855 & $0.880(0.365-2.121)$ & 0.776 & $2.842(0.857-9.424)$ & 0.088 \\
\hline$<3.38 \mathrm{mIU} / \mathrm{mL}$ & $1.427(0.702-2.901)$ & 0.326 & $1.195(0.590-2.420)$ & 0.622 & $2.047(0.963-4.351)$ & 0.063 & $0.731(0.361-1.482)$ & 0.385 & $0.764(0.359-1.628)$ & 0.485 \\
\hline $\begin{array}{c}\geq 3.38 \mathrm{mIU} / \mathrm{mL} \\
\mathrm{E} 2\end{array}$ & $0.713(0.370-1.374)$ & 0.312 & $0.540(0.284-1.025)$ & 0.060 & $1.707(0.883-3.298)$ & 0.112 & $0.802(0.421-1.527)$ & 0.502 & $1.311(0.642-2.677)$ & 0.457 \\
\hline$<26.00 \mathrm{pg} / \mathrm{mL}$ & $1.439(0.720-2.880)$ & 0.303 & $0.685(0.342-1.372)$ & 0.285 & $2.322(1.134-4.755)$ & 0.021 & $0.510(0.247-1.050)$ & 0.068 & $0.874(0.400-1.911)$ & 0.736 \\
\hline$\geq 26.00 \mathrm{pg} / \mathrm{mL}$ & $0.795(0.404-1.563)$ & 0.506 & $0.840(0.429-1.647)$ & 0.612 & $1.821(0.905-3.664)$ & 0.093 & $0.506(0.256-1.002)$ & 0.051 & $1.604(0.785-3.280)$ & 0.195 \\
\hline
\end{tabular}

Note: AOR, adjusted odds ratio; 95\% CI, 95\% confidence interval; BMI, body mass index; PLT, platelet count; PT, prothrombin time; aPTT, activated par tial thromboplastin time; FSH follicle stimulating hormone; LH, luteinizing hormone; E2, estradiol. The adjusted odds ratio on the basis of risk factors, such as age, BMI, PLT, PT, aPTT, FSH, LH, and E2. 
Since clinical factors, including aPTT, PAI-1, PT, and homocysteine, have been associated with RPL, we investigated the association of these clinical variables with GP6 gene variants in RPL patients who had suffered three or more pregnancy losses. We found that aPTT, PAI-1, PT, homocysteine, and PAI-1 levels were significantly associated with these RPL patients (Supplementary Tables S6-S8). Furthermore, Figure 1 depicts the analysis of variance for aPTT, PAI-1, PT, and homocysteine levels according to GP6 polymorphisms (RPL $\geq 3$ ). Our association data are statistically quite weak when comparing to the normal genome-wide association study (GWAS) significance threshold of $5 \times 10^{-8}$.

(A)

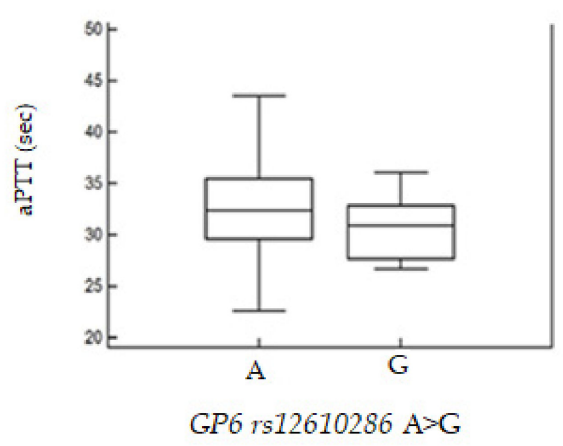

(C)

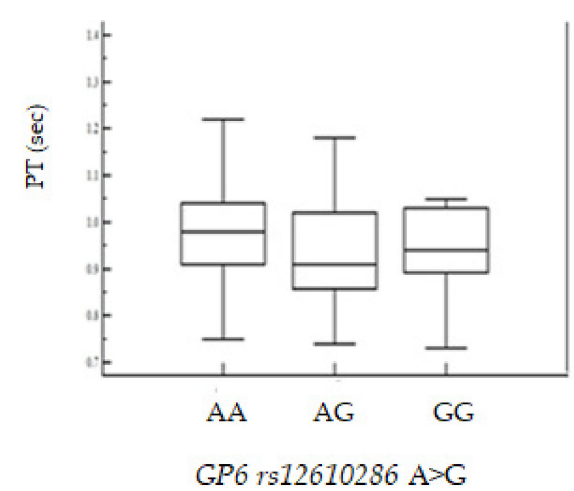

(B)

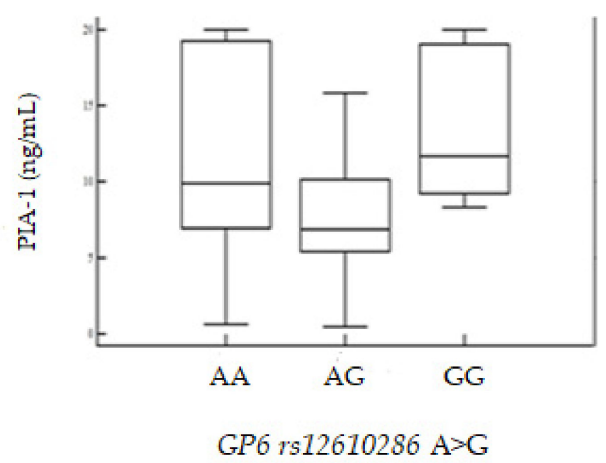

(D)

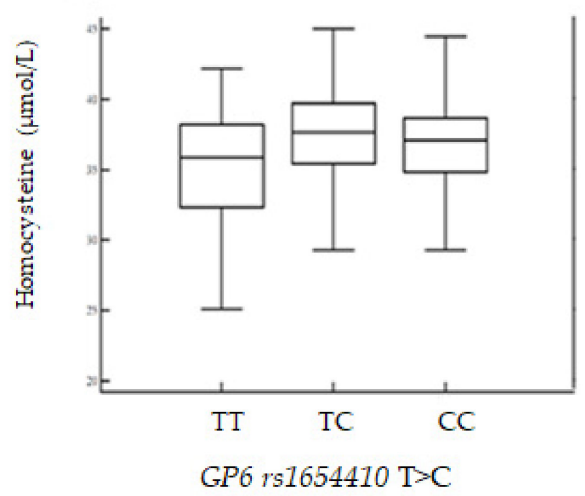

Figure 1. Analysis of variance for aPTT, PAI-1, PT, and homocysteine levels according to GP6 polymorphisms $(\mathrm{PL} \geq 3)$. (A) aPTT levels were significantly different $(p=0.047)$ between GP6 rs12610286 AA (mean \pm $\mathrm{SD}, 32.36 \pm 4.30), \mathrm{AG}(32.73 \pm 3.99)$, and GG (30.49 \pm 3.02$)$. (B) PAI-1 levels were significantly different $(p=0.0003)$ between GP6 rs12610286 AA (mean \pm SD, $11.75 \pm 6.01)$, AG (7.89 \pm 4.34$)$, and GG (13.26 \pm 4.88). (C) PT (sec) levels were significantly different $(p=0.045)$ between GP6 rs12610286 AA (mean \pm SD, $0.97 \pm 0.10), \mathrm{AG}(0.94 \pm 0.12)$, and GG (0.95 \pm 0.09$)$. (D) Homocysteine levels were significantly different $(p=0.026)$ between GP6 rs1654410 TT (mean \pm SD, $36.54 \pm 3.54)$, TC (37.12 \pm 3.88$)$, and CC (35.04 \pm 4.31$)$.

\section{Discussion}

In this study, we selected five GP6 polymorphisms (rs1654410 T>C, rs1671153 T>G, rs1654419 $\mathrm{G}>\mathrm{A}$, rs12610286 A>G, and rs1654431 G>A) that were candidate biomarkers for RPL risk.

Then, we investigated the association between these polymorphisms and RPL prevalence. We found that GP6 polymorphisms were associated with an increased risk of RPL and that the GP6 haplotypes rs1654410 T>C, rs1671153 T>G, rs1654419 G>A, rs12610286 A>G, and rs1654431 G>A occurred significantly more frequently in women with RPL. The haplotype analysis of the five genetic markers revealed that five haplotypes (GP6 rs1654410 T>C, rs1671153 T>G, rs1654419 G>A, rs12610286 A>G, and rs1654431 G>A; [ $p<0.001$; FDR-corrected $p<0.001$ OR, 19.13; 95\% CI, 1.142-320.200; $p=0.0002$; FDR-corrected $p=0.001$ ] were significantly associated with increased RPL susceptibility.

Many GP6 polymorphisms have been identified, and previous studies have demonstrated that genetic changes in GP6 have an effect on platelet function. Platelets are small, acellular cells that aggregate 
to form blood clots on blood vessels and are essential for hemostasis. As a platelet membrane protein, GP6 is generally accepted to be produced by platelet activation, adhesion, and aggregation. Interestingly, the importance of the genetic diversity of the GP6 gene for platelet aggregation was highlighted by a genome-wide meta-analysis conducted by Johnson et al. [20]. Moreover, a previous study identified several SNPs (rs1671153, rs1654419, and rs1613662) in patients with sticky platelet syndrome (SPS) [21] and a history of miscarriage (rs1671152, rs1654433, rs1613662, rs1654416, and rs2304167) [22]. In addition to their normal role in hemostasis, platelets can cause arterial thrombosis by blocking the collateral arteries after a collagen-rich atherosclerotic plaque has ruptured, resulting in heart attack and stroke [23]. After an atherosclerotic plaque rupture, the relationship between GP6 and thrombus GP6-specific signaling plays a major role in platelet adhesion to activated endothelium [24,25] and thrombus formation [26], whereas platelet GP6 mediates adherence to the endothelial matrix [27].

GP6 is a receptor that contains an immunoglobin-like domain, is structurally and functionally similar to an immunoreceptor, and is exclusively expressed in platelets and platelet precursors called megakaryocytes. Although it is unknown how thrombotic formation through GP6 can occur so rapidly, it is known that various agonists (collagen, thrombin, and ADP) activate GP6 on the platelet membrane to induce binding to fibrinogen and consequently promote the aggregation of platelets and granule release [28,29].

In addition, collagen activates platelets by promoting the phosphorylation of phospholipase C- $\gamma 2$ (PLC- $\gamma 2$ ) through GP6 [30]. Platelets that circulate along the bloodstream respond to collagen that is exposed at the wound site on the vessel wall via GP6 recognition and signaling. Collagen is a typical extracellular matrix that is used to form a thrombus, which is exposed to the bloodstream when a wound on the blood vessel wall develops, thus forming a platelet-attachable surface in the artery and acting as the main stimulant for platelet activation. Therefore, achieving a better understanding of this process may lead to the development of treatment modalities that inhibit or prevent thrombus formation. We predict that these mechanisms will be important in RPL patients based on the association with GP6.

GP6 dimers recognize collagen, and a portion of GP6 is present as a dimer in resting platelets. Upon platelet activation, GP6 dimer levels increase [31,32]. GP6 forms a noncovalent bond with platelet glycoprotein $\mathrm{Ib} \alpha(\mathrm{GPIb} \alpha)$ on activated platelets and signals through the associated proto-oncogene tyrosine-protein kinase Src (Src)/spleen tyrosine kinase (Syk) kinase pathway. Crucially important in the GP6-mediated signal is the presence of an immunoreceptor tyrosine-based activation motif (ITAM) [33-35] in the cytoplasmic domain Fc receptor common $\gamma$ signaling chain (FcR $\gamma$ ). Interestingly, GP6 signaling not only regulates normal hemostasis but also contributes to cardiovascular disease, inflammation, coagulation control, and tumor metastasis [36-41].

Currently, there are some reported associations between GP6 and platelet syndrome in RPL [16,34,42,43].

In the present study, polymorphic GP6 genotype frequencies rose significantly as the frequency of pregnancy loss increased. Moreover, GP6 genotypes and haplotypes were associated with known contributors to increased blood coagulation, including elevated plasma PAI-1 and BMI, lower PT and aPTT, and plasma concentrations of vascular risk factors (homocysteine, FSH, and total cholesterol) in RPL. We anticipate that these GP6 genotype combinations contribute to the incidence of RPL in Korean women. Alterations in the fibrinolysis cascade that cause hypo-fibrinization or hyperfibrinolysis may interfere with the placenta and result in poor pregnancy outcomes. Defects in this process can negatively affect trophoblast transplantation as well as the placenta and may result in RPL. Moreover, higher PLT indices have been reported to increase the risk of thrombosis. Previous reports also suggest that hormones, including FSH, LH, and E2 are involved in RPL, since FSH, LH, and E2 levels were elevated in the RPL group compared with the control group. The control group consisted of women who had abortions with known causes, such as uterine septum and parental chromosomal abnormalities. However, in hemostasis, factor V Leiden mutation, prothrombin G20210A variant, the decreased activity of antithrombin, protein C, protein S, the increased coagulation factor VIII activity, dysfibrinogenaemia, fibrinolysis abnormalities, antiphospholipid syndrome, and the detection of plasma levels of substances released from platelets factors related to thrombophilia should also be investigated. 
Reports on the relationship between GP6 and pregnancy loss are very limited. In addition, the biological function of GP6 pregnancy is not well known. Genetic mutations in the blood coagulation factor cause a prethrombotic condition through deficiencies of blood coagulation inhibitors, the overproduction of pre-coagulation proteins, abnormalities in fibrinolysis, and damage to the vascular endothelium. As the diagnosis of neonatal thromboembolism increases, the role of genetic risk factors becomes more important.

GP6 is a platelet transmembrane glycoprotein that plays a significant role in collagen-initiated signal transduction and platelet pro-coagulant activity; therefore, the observed variation in the GP6 gene region may influence risk for thromboembolic disorders [44].

A previous report shows that a significantly higher occurrence of mutant genotypes of GP6 SNPs, namely rs1671153, rs1654410, rs1654419, and rs1613662, in recurrent miscarriages (RM) cases, suggesting a risk association for pregnancy loss [45]. Another study has shown risk associations of mutant genotypes at rs1671153, rs1654419, and rs1613662 SNPs with thrombotic disorders [46].

Patients of pregnancy loss demonstrate reduced platelet function [47]. The GP6 plays a critical role in platelet adhesion and activation, and GP6 has a major role in collagen-induced platelet signaling [45]. It is thought that changes in platelet adhesion and activation by GP6 polymorphism (rs1654410T $>$ C, rs1654419 G>A, and rs1654431 G>A) would have influenced platelet reactivity toward collagen and therefore influence platelet function and the maintenance of pregnancy.

Based on the present study, we suggest that the genotype combinations of GP6 polymorphisms rs1654410/GP6 rs1671153 (CC/TT) may contribute to the diagnosis of RPL in Korean women. Therefore, additional studies are needed to clarify the association between GP6 polymorphisms and RPL.

MicroRNAs (miRNAs) are associated with platelet reactivity; however, there is a lack of information regarding the role of miRNAs in megakaryocyte signaling cascades, and miRNAs are not known to regulate collagen-induced GP6 signaling. One previous report bioinformatically predicted that miR-15a-5p targets were expressed in megakaryocytes and subsequently enriched target genes that are also known to be downstream targets of platelet GP6 signaling [48]. These findings indicate that miR-15a-5p regulates the potential master-miRNA GP6-mediated megakaryocyte signaling and platelet activation. Therefore, the present study provides a basis for future research associated with miRNA in RPL patients.

It is not sufficient to make recommendations for RPL patient management based on our research results alone. Our results indicate that several polymorphisms are associated with clinical variables in RPL patients: GP6 rs1671153 T>G, rs1654419 G>A, and rs12610286 A>G were associated with higher homocysteine levels, elevated creatinine levels, and PAI-1, respectively. Therefore, GP6 polymorphisms may contribute to RPL and are potential biomarkers for assessing RPL risk. However, our study does have various limitations, which are outlined in the Discussion, and efforts to overcome these problems are necessary. Our chosen method of liquid biopsy has been recently investigated in depth, and we therefore plan to continue research to prevent platelet-related diseases by analyzing GP6-related microRNA, non-coding RNAs, and exosomes; in this regard, the present study provides a basis for future research associated with miRNA in RPL patients.

Although we found an association between GP6 polymorphisms and RPL, there are some limitations to our study: lack of a survey for vascular risk factors, clinical insufficiency from control participants, and the relatively small sample size of the control and RPL group, which highlights the need for additional studies in larger patient populations. Mechanism by which GP6 polymorphisms affect the onset of RPL remains unclear. Additional information regarding risk factors for RPL patients is lacking, and additional research is needed. The population of this study was restricted to Korean patients.

\section{Conclusions}

In conclusion, we identified associations between GP6 gene polymorphisms (rs1654410 T>C, rs1671153 T>G, rs1654419 G>A, rs12610286 A>G, and rs1654431 G>A) and RPL prevalence in Korean women. Our findings suggest that GP6 polymorphisms may contribute to RPL and are potential biomarkers for assessing RPL risk. For example, several polymorphisms were associated with clinical 
variables in RPL patients: GP6 rs1671153 T>G, rs1654419 G>A, and rs12610286 A>G were associated with higher homocysteine levels, elevated creatinine levels, and PAI-1, respectively.

Moreover, the haplotype frequencies of GP6 rs1654410 T>C, rs1671153 T>G, rs1654419 G>A, rs12610286 A>G, and rs1654431 G>A were significantly different between RPL patients and control participants. Together, these results highlight the need for large and heterogeneous genetic studies to confirm the present findings and to validate potential biomarkers of RPL for prevention and prognosis.

Supplementary Materials: The following are available online at http://www.mdpi.com/2073-4425/11/8/862/s1. Figure S1. Linkage disequilibrium (LD) patterns of GP6 SNPs. Values in squares are LD between single markers. There were no strong LDs between loci rs1654410C $>$ T, rs1671153 T>G, rs1654419 G>A, rs12610286 A>G and rs1654431 G> in RPL subjects. Table S1: Information of GP6 gene polymorphisms for PCR-RFLP and real-time PCR analysis. Table S2: Haplotype analysis of GP6 gene polymorphisms in RPL and controls. Table S3: Genotype combination for the GP6 polymorphisms in recurrent pregnancy loss (RPL) patients and control subjects. Tables S4-S5: Haplotype analysis of GP6 gene polymorphisms in RPL and controls. Tables S6-S8: Differences of various clinical parameters according to GP6 gene polymorphisms in RPL. Table S9: Frequencies of GP6 polymorphisms in world-wide populations.

Author Contributions: H.J.A. and E.H.A. conceived and designed the experiments. J.O.K., C.S.R. and H.S.P. performed the experiments. H.J.A., H.S.P., C.S.R. and N.K.K. analyzed the data. S.H.C., J.H.K., W.S.L. and J.R.L. contributed reagents/materials/analysis tools. H.J.A., E.H.A. and N.K.K. wrote the paper. The article was edited by Y.R.K. and N.K.K. All authors have read and agreed to the published version of the manuscript.

Funding: This study was partially supported by a grant from the Korea Healthcare technology R\&D Project, Ministry for Health, Welfare \& Family Affairs, Republic of Korea (HI18C19990200), and by the National Research Foundation of Korea (NRF) funded by the Ministry of Education, Science and Technology (NRF-2017R1D1A1B03031542, 2018R1D1A1A09082764, 2018R1D1 A1B07044096, NRF-2020R1I1A1A01072008).

Conflicts of Interest: The authors have no financial conflicts of interest.

\section{References}

1. Coulam, C.B.; Clark, D.A.; Beer, A.E.; Kutteh, W.H.; Silver, R.; Kwak, J.; Stephenson, M. Current clinical options for diagnosis and treatment of recurrent spontaneous abortion. Clinical Guidelines Recommendation Committee for Diagnosis and Treatment of Recurrent Spontaneous Abortion. Am. J. Reprod. Immunol. 1997, 38, 57-74. [CrossRef] [PubMed]

2. Rai, R.; Regan, L. Recurrent miscarriage. Lancet 2006, 368, 601-611. [CrossRef]

3. Sierra, S.; Stephenson, M. Genetics of recurrent pregnancy loss. Semin. Reprod. Med. 2006, 24, 17-24. [CrossRef]

4. Clemetson, K.J.; Clemetson, J.M. Platelet collagen receptors. Thromb. Haemost. 2001, 86, 189-197. [CrossRef] [PubMed]

5. Clemetson, J.M.; Polgar, J.; Magnenat, E.; Wells, T.N.; Clemetson, K.J. The platelet collagen receptor glycoprotein VI is a member of the immunoglobulin superfamily closely related to FcalphaR and the natural killer receptors. J. Biol. Chem. 1999, 274, 29019-29024. [CrossRef] [PubMed]

6. Primer3 Software. 2007. Available online: http://frodo.wi.mit.edu/primer3/input.htm (accessed on 1 July 2009).

7. Kubisz, P.; Ivankova, J.; Skerenova, M.; Stasko, J.; Holly, P. The prevalence of the platelet glycoprotein VI polymorphisms in patients with sticky platelet syndrome and ischemic stroke. Hematology 2012, 17, 355-362. [CrossRef]

8. Cole, V.J.; Staton, J.M.; Eikelboom, J.W.; Hankey, G.J.; Yi, Q.; Shen, Y.; Berndt, M.C.; Baker, R.I. Collagen platelet receptor polymorphisms integrin alpha2-beta1 C807 T and GPVI Q317L and risk of ischemic stroke. J. Thromb. Haemost. 2003, 1, 963-970. [CrossRef]

9. Sokol, J.; Biringer, K.; Skerenova, M.; Hasko, M.; Bartosova, L.; Stasko, J.; Danko, J.; Kubisz, P. Platelet aggregation abnormalities in patients with fetal losses: The GP6 gene polymorphism. Fertil. Steril. 2012, 98, 1170-1174. [CrossRef]

10. Yee, D.L.; Bray, P.F. Clinical and functional consequences of platelet membrane glycoprotein polymorphisms. Semin. Thromb. Hemost. 2004, 30, 591-600. [CrossRef]

11. Joutsi-Korhonen, L.; Smethurst, P.A.; Rankin, A.; Gray, E.; IJsseldijk, M.; Onley, C.M.; Watkins, N.A.; Williamson, L.M.; Goodall, A.H.; De Groot, P.G.; et al. The low-frequency allele of the platelet collagen signaling receptor glycoprotein VI is associated with reduced functional responses and expression. Blood 2003, 101, 4372-4379. [CrossRef] 
12. Rac, M.W.; Crawford, M.N.; Worley, K.C. Extensivethrombosisandfirst-trimesterpregnancylosscaused by sticky platelet syndrome. Obstet. Gynecol. 2011, 117, 501-503. [CrossRef] [PubMed]

13. Bick, R.L. Recurrent miscarriage syndrome due to blood coagulation protein/platelet defects: Prevalence, treatment and outcome results. DRW Metroplex Recurrent Miscarriage Syndrome Cooperative Group. Clin. Appl. Thromb. Hemost. 2000, 6, 115-125. [CrossRef] [PubMed]

14. Yagmur, E.; Bast, E.; Mühlfeld, A.S.; Koch, A.; Weiskirchen, R.; Tacke, F.; Neulen, J. High Prevalence of Sticky Platelet Syndrome in Patients with Infertility and Pregnancy Loss. J. Clin. Med. 2019, 8, 1328. [CrossRef] [PubMed]

15. Ryu, C.S.; Sakong, J.H.; Ahn, E.H.; Kim, J.O.; Ko, D.; Kim, J.H.; Lee, W.S.; Kim, N.K. Association study of the three functional polymorphisms (TAS2R46G $>$ A, OR4C16G $>$ A, and OR4 $\times 1 \mathrm{~A}>\mathrm{T}$ ) with recurrent pregnancy loss. Genes Genom. 2019, 41, 61-70. [CrossRef]

16. Hochberg, Y.; Benjamini, Y. More powerful procedures for multiple significance testing. Stat. Med. 1990, 9, 811-818. [CrossRef]

17. Ritchie, M.D.; Hahn, L.W.; Roodi, N.; Bailey, L.R.; Dupont, W.D.; Parl, F.F.; Moore, J.H. Multifactordimensionality reduction reveals high-order interactions among estrogen-metabolism genes in sporadic breast cancer. Am. J. Hum. Genet. 2001, 69, 138-147. [CrossRef]

18. Moore, J.H.; Williams, S.M. New strategies for identifying gene-gene interactions in hypertension. Ann. Med. 2002, 34, 88-95. [CrossRef]

19. Hahn, L.W.; Ritchie, M.D.; Moore, J.H. Multifactor dimensionality reduction software for detecting gene-gene and gene-environment interactions. Bioinformatics 2003, 19, 376-382. [CrossRef]

20. Johnson, A.D.; Yanek, L.R.; Chen, M.H.; Faraday, N.; Larson, M.G.; Tofler, G.; Lin, S.J.; Kraja, A.T.; Province, M.A.; Yang, Q.; et al. Genome-wide meta-analyses identifies seven loci associated with platelet aggregation in response to agonists. Nat. Genet. 2010, 42, 608-613. [CrossRef]

21. Kotulicova, D.; Chudy, P.; Skerenova, M.; Ivankova, J.; Dobrotova, M.; Kubisz, P. Variability of GP6 gene in patients with sticky platelet syndrome and deep venous thrombosis and/or pulmonary embolism. Blood Coagul. Fibrinolysis 2012, 23, 543-547. [CrossRef]

22. Sokol, J.; Skerenova, M.; Biringer, K.; Simurda, T.; Stasko, J. Glycoprotein VI gene variants affect pregnancy loss in patients with platelet hyperaggregability. Clin. Appl. Thromb. Hemost. 2018, 24, 202S-208S. [CrossRef] [PubMed]

23. Haining, E.J.; Matthews, A.L. Tetraspanin Tspan9 regulates platelet collagen receptor GPVI lateral diffusion and activation. Platelets 2017, 28, 629-642. [CrossRef] [PubMed]

24. Massberg, S.; Gawaz, M.; Grüner, S.; Schulte, V.; Konrad, I.; Zohlnhöfer, D.; Heinzmann, U.; Nieswandt, B. A crucial role of glycoprotein VI for platelet recruitment to the injured arterial wall in vivo. J. Exp. Med. 2003, 197, 41-49. [CrossRef] [PubMed]

25. Massberg, S.; Konrad, I.; Bültmann, A.; Schulz, C.; Münch, G.; Peluso, M.; Lorenz, M.; Schneider, S.; Besta, F.; Müller, I.; et al. Soluble glycoprotein VI dimer inhibits platelet adhesion and aggregation to the injuredvessel wall in vivo. FASEB J. 2004, 18, 397-399. [CrossRef]

26. Grothusen, C.; Umbreen, S.; Konrad, I.; Stellos, K.; Schulz, C.; Schmidt, B.; Kremmer, E.; Teebken, O.; Massberg, S.; Luchtefeld, M.; et al. EXP3179 inhibits collagen-dependent platelet activation via glycoprotein receptor-VI independent of AT1-receptor antagonism: Potential impact on atherothrombosis. Arterioscler. Thromb. Vasc. Biol. 2007, 27, 1184-1190. [CrossRef]

27. Schönberger, T.; Ziegler, M.; Borst, O.; Konrad, I.; Nieswandt, B.; Massberg, S.; Ochmann, C.; Jürgens, T.; Seizer, P.; Langer, H.; et al. The dimeric platelet collagen receptor GPVI-Fc reduces platelet adhesion to activated endothelium and preserves myocardial function after transient ischemia in mice. Am. J. Physiol. Cell Physiol. 2012, 303, C757-C766. [CrossRef]

28. Schwartz, S.M.; Heinmark, R.L.; Majesky, M.W. Developmental mechanisms underlying pathology of arteries. Physiol. Rev. 1990, 70, 1177-1209. [CrossRef]

29. Payrastre, B.; Missy, K.; Trumel, C.; Bodin, S.; Plantavid, M.; Chap, H. The integrin alpha IIb/beta 3 in human platelet signal transduction. Biochem. Pharmacol. 2000, 60, 1069-1074. [CrossRef]

30. Phillips, D.R.; Nannizzi-Alaimo, L.; Prasad, K.S. Beta3 tyrosine phosphorylation in alphaIIbbeta3 (platelet membrane GP IIb-IIIa) outside-in integrin signaling. Thromb. Haemost. 2001, 86, 246-258. 
31. Jung, S.M.; Moroi, M.; Soejima, K.; Nakagaki, T.; Miura, Y.; Berndt, M.C.; Gardiner, E.; Howes, J.-M.; Pugh, N.; Bihan, D.; et al. Constitutive dimerization of glycoprotein VI (GPVI) in resting platelets is essential for binding to collagen and activation in flowing blood. J. Biol. Chem. 2012, 287, 30000-30013. [CrossRef]

32. Loyau, S.; Dumont, B.; Ollivier, V.; Boulaftali, Y.; Feldman, L.; Ajzenberg, N.; Perrus, M.J. Platelet glycoprotein VI dimerization, an active process inducing receptor competence, is an indicator of platelet reactivity. Arterioscler. Thromb. Vasc. Biol. 2012, 32, 778-785. [CrossRef] [PubMed]

33. Moog, S.; Mangin, P.; Lenain, N.; Strassel, C.; Ravanat, C.; Schuhler, S.; Freund, M.; Santer, M.; Kahn, M.; Nieswandt, B.; et al. Platelet glycoprotein V binds to collagen and participates in platelet adhesion and aggregation. Blood 2001, 98, 1038-1046. [CrossRef] [PubMed]

34. Azorsa, D.O.; Moog, S.; Ravanat, C.; Schuhler, S.; Folléa, G.; Cazenave, J.P.; Lanza, F. Measurement of GPV released by activated platelets using a sensitive immunocapture ELISA-Its use to follow platelet storage in transfusion. Thromb. Haemost. 1999, 81, 131-138. [CrossRef] [PubMed]

35. Ravanat, C.; Freund, M.; Mangin, P.; Azorsa, D.O.; Schwartz, C.; Moog, S.; Dambach, J.; Cazenave, J.; Lanza, F.; Ravanat, C. GPV is a marker of in vivo platelet activation study in a rat thrombosis model. Thromb. Haemost. 2000, 83, 327-333.

36. Ombrello, C.; Block, R.; Morrell, C. Our expanding view of platelet functions and its clinical implications. J. Cardiovasc. Transl. Res. 2010, 3, 538-546. [CrossRef]

37. Riedl, J.; Pabinger, I.; Ay, C. Platelets in cancer and thrombosis. Hamostaseologie 2014, 34, 54-62. [CrossRef]

38. Versteeg, H.H.; Heemskerk, J.W.; Levi, M.; Reitsma, P.H. New fundamentals in hemostasis. Physiol. Rev. 2013, 93, 327-358. [CrossRef]

39. Zhang, W.; Huang, W.; Jing, F. Contribution of blood platelets to vascular pathology in Alzheimer's disease. J. Blood Med. 2013, 4, 141-147. [CrossRef]

40. Andrews, R.K.; Berndt, M.C.; Elalamy, I. Platelets: From function to dysfunction in essential thrombocythaemia. Eur. Haematol. Oncol. 2011, 7, 125-131. [CrossRef]

41. Sokol, J.; Skerenova, M.; Biringer, K.; Lasabova, Z.; Stasko, J.; Kubisz, P. Genetic variations of the GP6 regulatory region in patients with sticky platelet syndrome and Miscarriage. Expert Rev. Hematol. 2015, 8 , 863-868. [CrossRef]

42. Škereňová, M.; Sokol, J.; Biringer, K.; Ivanková, J.; Staško, J.; Kubisz, P.; Lasabova, Z. GP6 Haplotype of Missense Variants is Associated with Sticky Platelet Syndrome Manifested by Fetal Loss. Clin. Appl. Thromb. Hemost. 2018, 24, 63-69. [CrossRef] [PubMed]

43. Pijnenborg, R.; Vercruysse, L.; Hanssens, M. The uterine spiral arteries in human pregnancy: Facts and controversies. Placenta 2006, 27, 939-958. [CrossRef]

44. Urihata, K.; Kuniki, T.J. Characterization of human glycoprotein VI gene $5^{\prime}$ regulatory and promoter regions. Arterioscler. Thromb. Vasc. Biol. 2002, 22, 1733-1739. [CrossRef] [PubMed]

45. Siddesh, A.; Parveen, F.; Misra, M.K.; Phadke, S.R.; Agrawal, S. Platelet-specific collagen receptor glycoprotein VI gene variants affect recurrent pregnancy loss. Fertil. Steril. 2014, 2, 1078-1084. [CrossRef] [PubMed]

46. 1000 Genome Project Consortium; Abecasis, G.R.; Auton, A.; Brooks, L.D.; DePristo, M.A.; Durbin, R.M.; Handsaker, R.E. An integrated map of genetic variation from 1092 human genomes. Nature 2012, 491, 56-65. [CrossRef] [PubMed]

47. Dempsey, M.A.; Flood, K.; Burke, N.; Murray, A.; Cotter, B.; Mullers, S.; Dicker, P.; Fletcher, P.; Geary, M.; Kenny, D.; et al. Platelet function in patients with a history of unexplained recurrent miscarriage who subsequently miscarry again. Eur. J. Obstet. Gynecol. Reprod. Biol. 2015, 188, 61-65. [CrossRef]

48. Basak, I.; Bhatlekar, S.; Manne, B.K.; Stoller, M.; Hugo, S.; Kong, X.; Ma, L.; Rondina, M.T.; Weyrich, A.S.; Edelstein, L.C.; et al. miR-15a-5p regulates expression of multiple proteins in the megakaryocyte GPVI signaling pathway. J. Thromb. Haemost. 2019, 17, 511-524. [CrossRef]

(C) 2020 by the authors. Licensee MDPI, Basel, Switzerland. This article is an open access article distributed under the terms and conditions of the Creative Commons Attribution (CC BY) license (http://creativecommons.org/licenses/by/4.0/). 